\title{
Eletroless Silver Coated Nanocenosphere/Polymer Composite for EMI Shielding Effectiveness
}

\author{
Vynatheya $\mathbf{S}^{1 *}$, Bheema Raju V', Chandra Sagar L² and Sushma US ${ }^{1}$ \\ ${ }^{1}$ Department of Chemistry, Dr. Ambedkar Institute of Technology, Bangalore, India \\ ${ }^{2}$ Department of Mechanical Engineering, Dr Ambedkar Institute of Technology, Bangalore, India
}

\begin{abstract}
Electromagnetic interference (EMI) is an escalating concern in the modern electronic climate. As such it has become a critical area to consider when designing and packaging electronics. In this context, Electroless Silver Coated nanocenosphere (SCNC) in Poly Methyl Methacrylate (PMMA) and Acrylonitrile Butadiene Styrene (ABS) composites has been tried for its EMI shielding effectiveness. The strategy is to render the polymer conductive by dispersing SCNC in various proportions in these polymers separately and to promote wave absorption by making sheet of the polymer-based composite. In our study EDX (Energy Dispersive X-Ray) analysis confirmed the presence of Ag on the coated nano cenosphere. This was further, confirmed through Phase analysis by XRD (X-Ray Diffractometer) analysis. SEM (Scanning Electron Microscope) analysis was conducted to see the size, shape and adherence of the Ag coating on nanoenosphere particles and to observe the distribution of these particles in polymer matrix in the composite sheet. BET Surface area of electroless coated powders was quite high indicating good surface properties.

The processing methods, electrical properties (surface resistivity) and electromagnetic behavior (EMI shielding effectiveness) were investigated. Shielding effectiveness (SE) values of approximately $25 \mathrm{~dB}$ at a frequency of 1 $\mathrm{GHz}$ was obtained for coatings of Electroless Silver coated nanocenospheres in ABS polymer which was further increased by adding other conductive nano fillers (Graphene and multi walled Carbon Nano tube) along with coated nanocenospheres.
\end{abstract}

Keywords: Electroless coating; Electromagnetic interference; Nanocenospheres

\section{Introduction}

Electromagnetic Interference (EMI) is an important topic of interest in present times. As the technology is advancing, the importance to integrate large amount of electrical and electronic devices in various engineering fields such as automobiles, space, medical instruments etc. is of utmost need. In an electronic system, EMI can very badly affect the performance of an integrated circuit internally and also on the other electronic components which are in close proximity [1]. The interference sources may be internal or external to the electrical or electronic system and they may propagate by radiation or conduction. Commercially many devices are available for EMI shielding which are mostly metal based. The desire for high performance combined with reductions in size, weight and manufacturing cost suggests that polymer composites should be ideal materials for parts such as electronic instruments. Unfortunately polymer composites generally do not provide shielding from electromagnetic waves. However, if the polymer composites can be made conductive with additives they can be used as EMI shields in Electronics. Uniformly distributed fine conductive fillers in polymer matrix can be considered for EMI shielding. Composite sheets comprising of metal coated fly ash based nanocenospheres with other fine conductive additives dispersed in suitable polymer in optimum proportions are such materials which can be used as effective shielding materials.

The word Cenosphere literally means spherical particles which are hollow in structure. Cenospheres are fly ash based and belong to ceramic family with light weight, inert, hollow spheres which are typically comprising of silica and alumina and are filled with air or inert gas, mainly produced as a by-product of coal combustion unit at thermal power plants. Cenosphere particles are hard and rigid in nature; they are also water repellant and insulative. Cenospheres are very easy to handle and they easily flow and have a good surface area.
Due to their inert properties, they are not affected by water, acids, alkalis [2] or solvents.

There are many methods which are available to coat ceramics such as: Electro-plating, Metal Spraying, Vacuum metalizing, hot dipping, Electroless plating etc. Among all these methods, Electroless plating method is considered to be more suitable for the present study involving fine particles, because the substrates are in the form of powder (nanocenosphere) and the coating method provides a thin, homogenized [3] and controllable coating [4]. In the present study electroless Silver coated nanocenosphere [5] was successfully studied for EMI Shielding effectiveness.

\section{Experimental Section}

\section{Raw material and chemicals used}

Fly ash based Cenosphere particles which have been collected from Raichur Thermal Power station. Commercially available Silver Nitrate, Glucose, Poly Ethylene Glycol, Sodium Hydroxide, Ammonia solution, Poly Methyl Methacrylate (PMMA), Acrylo-Nitrile Butadiene Styrene (ABS), Dimethyl Formamide (DMF), Acetone, Chloroform, Tin Chloride, Palladium Chloride, Hydrochloric Acid, Nitric Acid and Sulphuric Acid have been used in our study.

*Corresponding author: Vynatheya S, Department of Chemistry, Dr. Ambedkar Institute of Technology, Bangalore, India, Tel: 09449088791; E-mail srinivasanvyn@rediffmail.com

Received January 01, 2018; Accepted January 25, 2018; Published February 05, 2018

Citation: Vynatheya S, Bheema RV, Chandra SL, Sushma US (2018) Eletroless Silver Coated Nanocenosphere/Polymer Composite for EMI Shielding Effectiveness. J Material Sci Eng 7: 420. doi: 10.4172/2169-0022.1000420

Copyright: (c) 2018 Vynatheya S, et al. This is an open-access article distributed under the terms of the Creative Commons Attribution License, which permits unrestricted use, distribution, and reproduction in any medium, provided the original author and source are credited. 


\section{Preparation of nanocenospheres}

About 50 gm. of acid (dil. $\mathrm{H}_{2} \mathrm{SO}_{4}$ ) washed cenosphere powder was taken in high energy ball mill with Zirconia jar containing Zirconia balls of different sizes as grinding media. To this powder $150 \mathrm{ml}$ of Acetone was added, and ground for $40 \mathrm{~h}$ with $300 \mathrm{RPM}$ speed and dried in an oven at $100^{\circ} \mathrm{C}$. The ground powder was measured for particle size. The average size of the particles after high energy ball milling was ranging between $100-250 \mathrm{~nm}$. Nanocenosphere thus obtained was again washed with dilute acid $\left(\mathrm{H}_{2} \mathrm{SO}_{4}\right)$ followed by filtration which was carried out using fine membrane filter and the residue was dried again. The BET surface area of the powder was measured and it was ranging from $225-275 \mathrm{~m}^{2} /$ gram indicating high surface area.

\section{Pre-treatment of nanocenospheres}

- The nanocenosphere powder thus prepared was washed well before carrying out the coating process in order to remove impurities. The following process was adopted for acid wash: About $50 \mathrm{~g}$ of nanocenosphere was taken in a beaker with a solution of Sulphuric acid of $0.5 \%$ concentration $(500 \mathrm{ml})$. The fine material in acid was thoroughly agitated using magnetic stirrer for about one hour and filtered using fine filter paper. The residue was then dried in a hot air oven at $90^{\circ} \mathrm{C}$.These acid washed nanocenosphere particles are used for Silver coating after following pretreatments which are to be carried out prior to electroless plating.

- The nanocenosphere thus prepared was transferred to sensitization bath compositions shown below and stirred each time with a mechanical stirrer.

- Sensitization bath: $5 \mathrm{~g} / \mathrm{l} \mathrm{SnCl}_{2}$ and $30 \mathrm{ml} / \mathrm{l}$ conc. $\mathrm{HCl}$ for $1 \mathrm{hr}$.

- Activation bath: $0.1 \mathrm{~g} / \mathrm{l} \mathrm{PdCl}, 5 \mathrm{ml} / \mathrm{l}$ conc. $\mathrm{HNO}_{3}$ and $25 \mathrm{ml} / \mathrm{l}$ conc. $\mathrm{HCl}-1 \mathrm{~h}$.

- After each step the treated nanocenosphere powder was filtered and washed with deionized water followed by drying at $110^{\circ} \mathrm{C}$ in an oven. The washing after each step was essential to remove the chemicals used in the plating process so that only the Silver coated nanocenosphere is obtained.

\section{Mechanism of electroless silver coating}

The Eletroless Silver coating bath consists of the following components:

- $10 \mathrm{~g}$ of Silver Nitrate taken in $400 \mathrm{ml}$ of water and $3 \mathrm{~g}$ of $\mathrm{NaOH}$ is added to the solution followed by addition of Ammonia solution drop by drop until the solution becomes transparent. The final solution is made up to $500 \mathrm{ml}$. Here Silver nitrate is used as the source of Silver ions. Separately reducing agent was prepared by dissolving a mixture of $25 \mathrm{~g}$ of Glucose, 20 $\mathrm{ml}$ of Ethyl alcohol and $40 \mathrm{ml}$ of Glycol in $500 \mathrm{ml}$ of water. The coating bath is prepared by mixing both the above solutions with continuous stirring for an hour. The $\mathrm{pH}$ of the solution is maintained at around 7-8 by continuous monitoring with a $\mathrm{pH}$ meter.

\section{Preparation of SCNC/PMMA composite sheet}

Preparation of SCNC/PMMA composite sheet was carried out by dissolving PMMA in Chloroform followed by adding SCNC with constant stirring for an hour followed by sonication at high frequency. The concentrated polymer solution with uniformly dispersed Silver coated nanocenosphere (SCNC) was carefully transferred to a petri dish and allowed to dry overnight. The composite sheet thus obtained was tested for Shielding effectiveness.

The detailed procedure of SCNC/PMMA composite sheet (30\% Silver coated nanocenosphere) preparation is as described below:

$7 \mathrm{~g}$ of PMMA was added in $100 \mathrm{ml}$ Chloroform and stirred with slight heating until PMMA dissolved completely in Chloroform. After complete dissolving of PMMA, $3 \mathrm{~g}$ of Silver coated Nanocenosphere was added to the above polymer solution and again stirred well for an hour. Finally the reaction mixture was ultra sonicated for half an hour to get uniform dispersion of the coated powder in polymer matrix to get good connectivity between the particles, followed by pouring solution on to a petri dish and dried in a vacuum oven at $100^{\circ} \mathrm{C}$. The petri dish was taken out after cooling and the dried sheet was carefully removed. Vacuum drying helped in preventing air bubble formation during drying thereby improving the conductivity of the sheet. In a similar way different weight percentages of SCNC/PMMA composites sheets were prepared ranging from $5 \%$ to $35 \%$.

\section{Preparation of SCNC/ABS composite sheet}

Preparation of SCNC/ABS composite sheet was carried out by dissolving ABS in DMF and adding SCNC with constant stirring for an hour followed by sonication. The concentrated polymer solution with uniformly dispersed SCNC was carefully transferred to a petri dish and Vacuum dried. Different weight percentages of SCNC/ABS composite sheets were prepared as in the case of PMMA polymer.

\section{Characterization}

Both nanocenospheres (NC) and Silver coated nanocenospheres (SCNC) were subjected to XRD, EDAX and SEM analysis. The presence of the element Ag was confirmed both by XRD and EDAX tests in SCNC indicating that even after repeated washing the required coated metal was present on the particle surface.

The XRD patterns of nanocenosphere (NC) and Silver coated nanocenosphere (SCNC) showed glassy nature which is inherent property of fly ash based cenosphere. However, SCNC pattern was more crystalline after coating of silver (Figures 1 and 2).

The coated and uncoated nanocenosphere powders were characterized for chemical composition by Energy Dispersive X Ray method (EDAX). Figures 3 and 4 give the composition of the respective powders. It can be noted here that Silver is present on the surface of

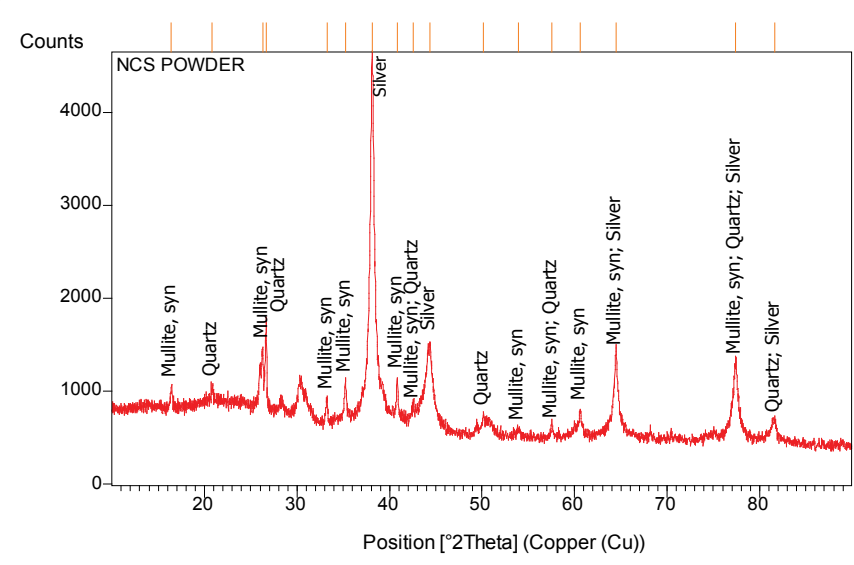

Figure 1: XRD of SCNC. 
Citation: Vynatheya S, Bheema RV, Chandra SL, Sushma US (2018) Eletroless Silver Coated Nanocenosphere/Polymer Composite for EMI Shielding Effectiveness. J Material Sci Eng 7: 420. doi: 10.4172/2169-0022.1000420

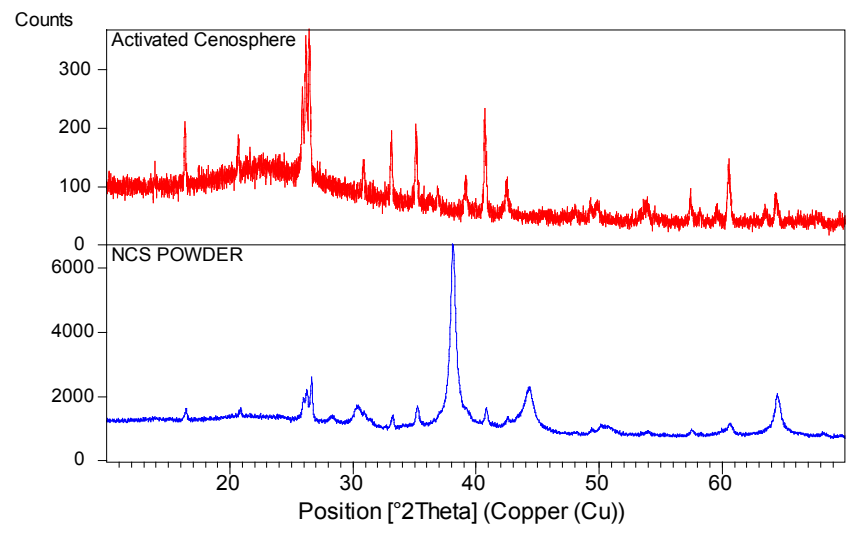

Figure 2: Comparison of XRD of NC and SCNC.

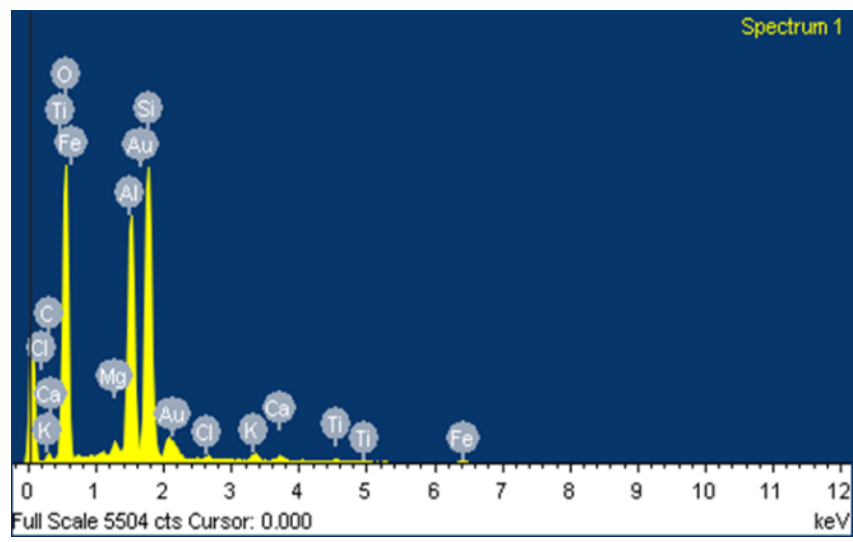

Figure 3: EDAX of nanocenosphere.

\begin{tabular}{|c|c|c|}
\hline Element & Weight\% & Atomic\% \\
\hline C K & 0.00 & 0.00 \\
O K & 68.13 & 79.02 \\
Mg K & 0.80 & 0.61 \\
Al K & 12.36 & 8.47 \\
Si K & 16.83 & 11.12 \\
Cl K & 0.00 & 0.00 \\
K K & 0.57 & 0.27 \\
Ca K & 0.48 & 0.22 \\
Ti K & 0.28 & 0.11 \\
Fe K & 0.55 & 0.18 \\
Totals & 100.00 & \\
\hline
\end{tabular}

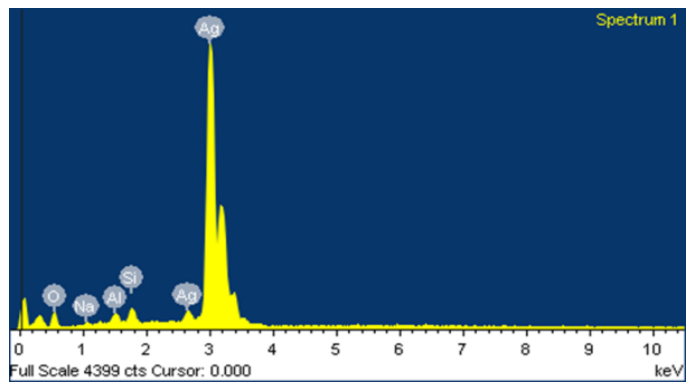

Figure 4: EDAX of coated nanocenosphere. coated nanocenosphere where as it is absent in uncoated powder confirming successful coating of silver by electroless process even after repeated distilled water washings of the coated powder.

The SEM micrographs were taken at different magnifications. At low magnification of $1.0 \mathrm{KX}$ (Figure 5) the morphology of the coated powders were found to be almost uniform. However, the particles could not be dispersed because of high agglomeration and very good attachment between fine particles. Further, at high magnification of 5.0 KX (Figure 6) though agglomeration of particles was observed, the particle size of individual grains was measured to be ranging between 100-150 nm (Figures 7-9).

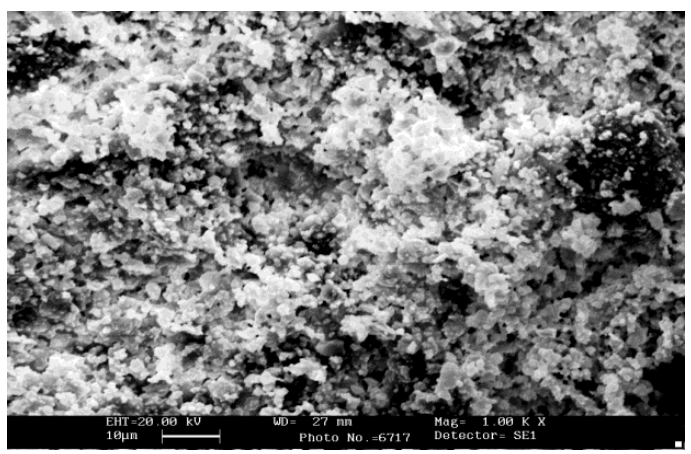

Figure 5: SEM micrograph of SCNC at $1.0 \mathrm{KX}$ showing highly agglomerated coated particles.

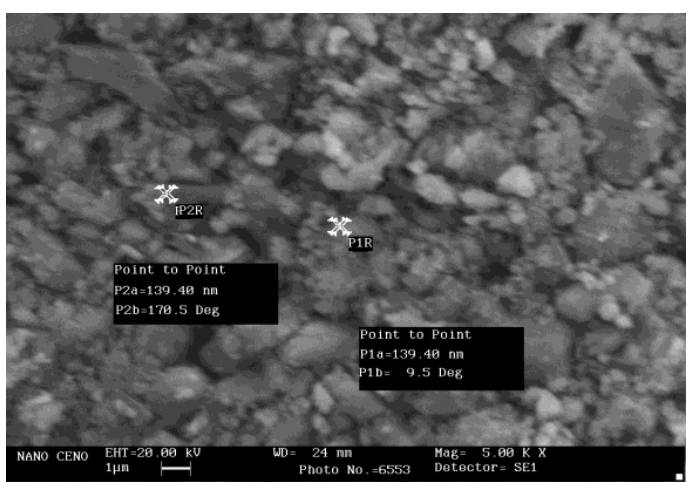

Figure 6: SEM micrograph of SCNC at $5.0 \mathrm{KX}$ showing aggolomerated coated particles.

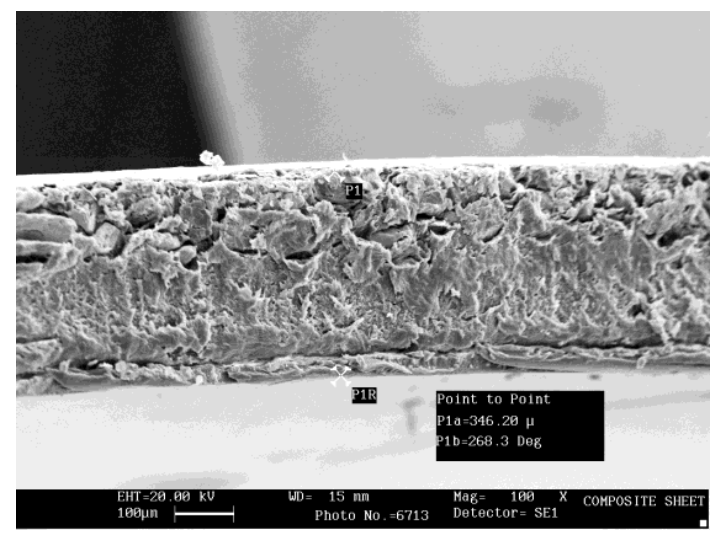

Figure 7: SEM micrograph of cross sectional view of composite sheet at $100 \mathrm{X}$ ( $30 \%$ SCNC) showing uniform distribution of SCNC particles in ABS polymer matrix exposed at lower magnification. Thickness of sheet was around $0.5 \mathrm{~mm}$. 


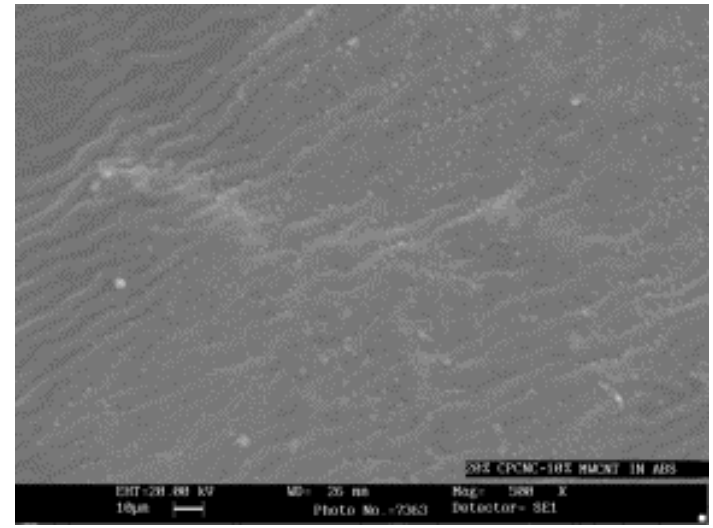

Figure 8: SEM micrograph of ABS composite sheet at $500 \times(30 \%$ SCNC) gives distribution of $30 \%$ SCNC particles in ABS polymer matrix. Here a uniform wave like structure can be observed. This uniform distribution can be attributed to higher conductivity in the case of $30 \%$ SCNC composite sheet.

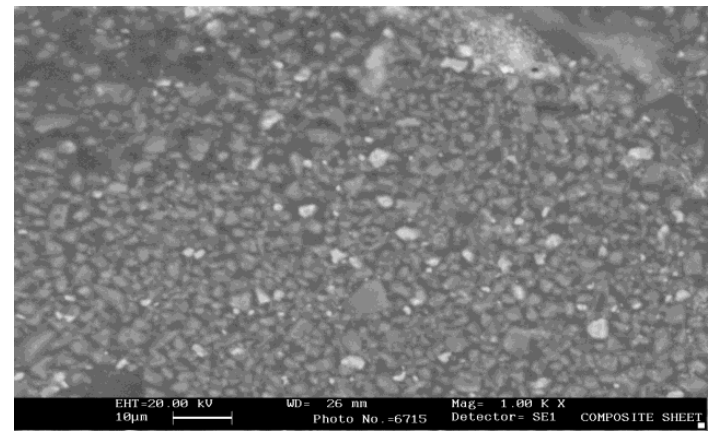

Figure 9: SEM micrograph of PMMA composite sheet at $1.0 \mathrm{KX}(30 \% \mathrm{SCNC})$ However, the distribution is not so uniform.

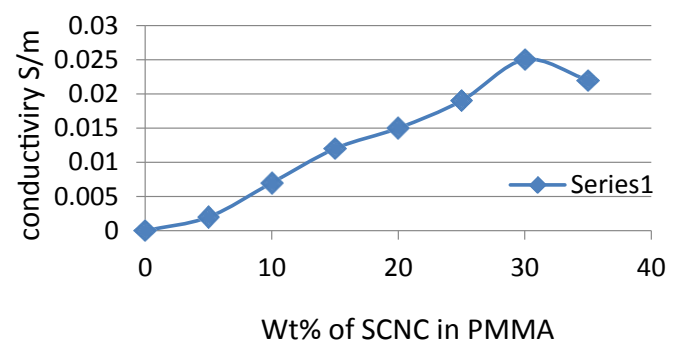

Figure 10: Conductivity of PMMA composite sheet for different wt $\%$ of SCNC.

\section{Results and Discussion}

The SCNC powder was subjected to surface area measurements before and after electroless coating. The BET surface area was measured to be around $250 \mathrm{~m}^{2} / \mathrm{g}$.

The surface area was found to be quite significant which means that the powder has good surface characteristics like higher surface energy and surface reactivity.

Resistivity of the composite sheet after dispersion of SCNC in the polymer matrix: The resistivity of the composite sheet was measured by four probe technique and it was ranging between 40 to $200 \mathrm{ohms} \mathrm{cm}$ at different $w \mathrm{t} \%$ of SCNC in polymer matrix and filler added SCNC in polymer matrix. This low resistivity indicates that the composite sheet has reasonably good conductivity which is about $0.025 \mathrm{~S} / \mathrm{m}$ for $30 \%$ composition and suitable for lower range EMI shielding applications and the conductivity v/s wt\% graph is as shown in the Figure 10 for PMMA and Figure 11 for ABS based composite sheet.

As a result of lower uniformity in distribution of SCNC particles (30 \%) in PMMA there is slight decrease in Conductivity of the composite sheet (Figure 10) than that of ABS composite sheet (30\%SCNC) which is about $0.028 \mathrm{~S} / \mathrm{m}$.

The conductivity of SCNC particles in ABS matrix (Figure 11) was higher than in the case of PMMA polymer due to higher uniformity of distribution of the particles in the composite sheet. Thus the EMI shielding effectiveness is higher in the case of SCNC distributed ABS composite sheet than that of PMMA composite sheet (Figures 12 and 13). Here also 30\% composition was taken for EMI studies as this composition has highest conductivity. It was observed from EMI measurements for SCNC composite sheets with PMMA and ABS composite sheets that both showed maximum effectiveness for $30 \%$ composition at a $1 \mathrm{GHz}$ frequency. However, the effectiveness was slightly higher for ABS composite which is due to higher conductivity.

Samples were also prepared with Silver coated nano-cenospheres along with conductive Nano fillers (Graphene/MWCNT) to study the Shielding effectiveness (SE). The overall Percentage of SCNC with conductive nano fillers was maintained at $30 \%$. The conductivity of the resultant composite sheet with $15 \%$ each of SCNC + Graphene/MWCNT was found to be higher than SCNC. Further, approximately $35 \mathrm{~dB}$ of SE was obtained for ABS filled with Silver coated nanocenospheres along with Nano conductive fillers (Figures 14 and 15).

\section{Conclusions}

- Eletroless method has been successfully adopted for coating Silver on nanocenospheres particles which can be observed through SEM, XRD and EDX.

- Uniformity of particles distribution in ABS matrix is good compared to that of PMMA matrix.

- Particle size of nanocenosphere particles was found to be in the range of 100-250 $\mathrm{nm}$ and the BET surface area was about $250 \mathrm{~m}^{2} / \mathrm{g}$.

- Conductivity was found to be $0.028 \mathrm{~S} / \mathrm{m}$ for $30 \mathrm{wt} \%$ of SCNC/ ABS composite. Further improvement in conductivity was observed by addition of conducting Nano fillers along with SCNC in the composite sheet there by increasing the shielding effectiveness to about $35 \mathrm{~dB}$ at a frequency of $1 \mathrm{GHz}$.

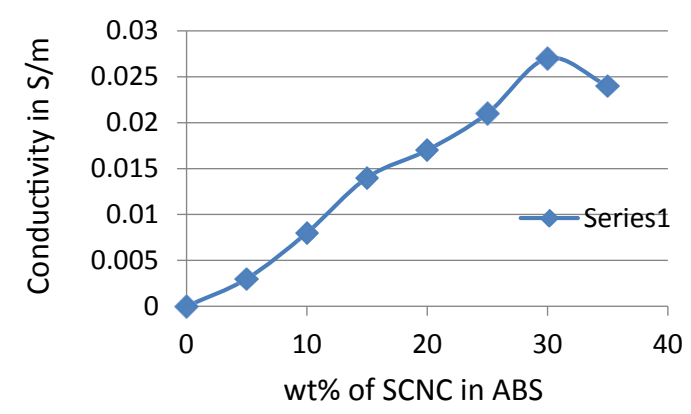

Figure 11: Conductivity of ABS composite sheet for different wt $\%$ of SCNC. 
Citation: Vynatheya S, Bheema RV, Chandra SL, Sushma US (2018) Eletroless Silver Coated Nanocenosphere/Polymer Composite for EMI Shielding Effectiveness. J Material Sci Eng 7: 420. doi: 10.4172/2169-0022.1000420

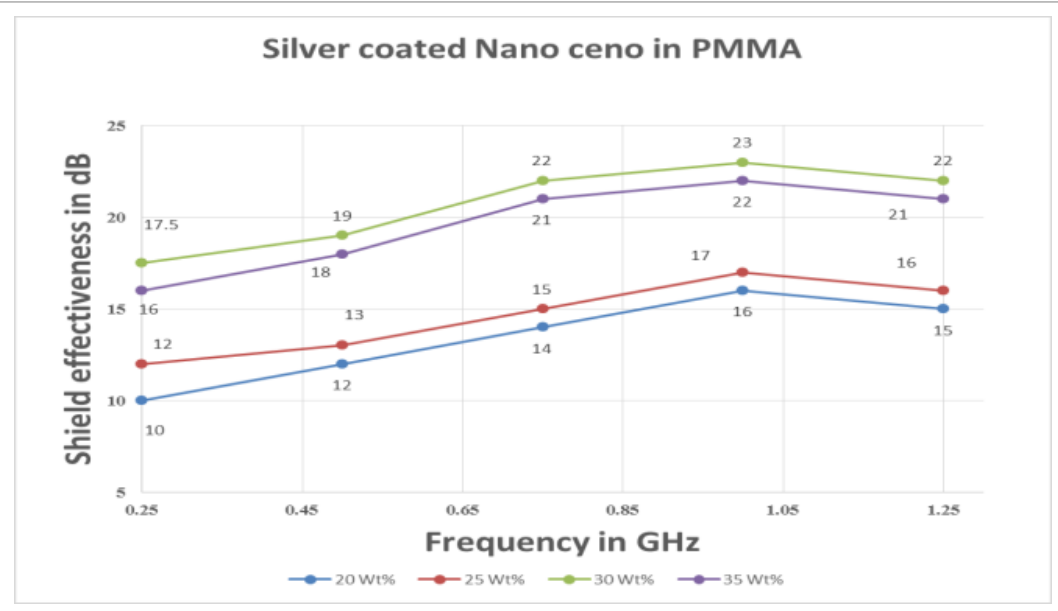

Figure 12: EMI Measurements for SCNC/PMMA Composite.

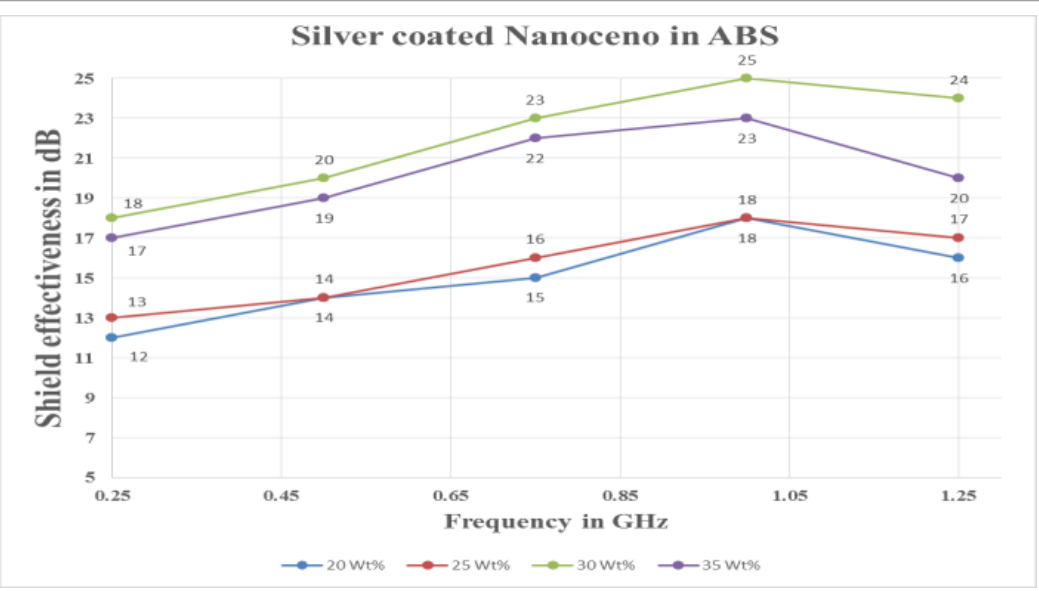

Figure 13: EMI Measurements for SCNC/ABS Composite.

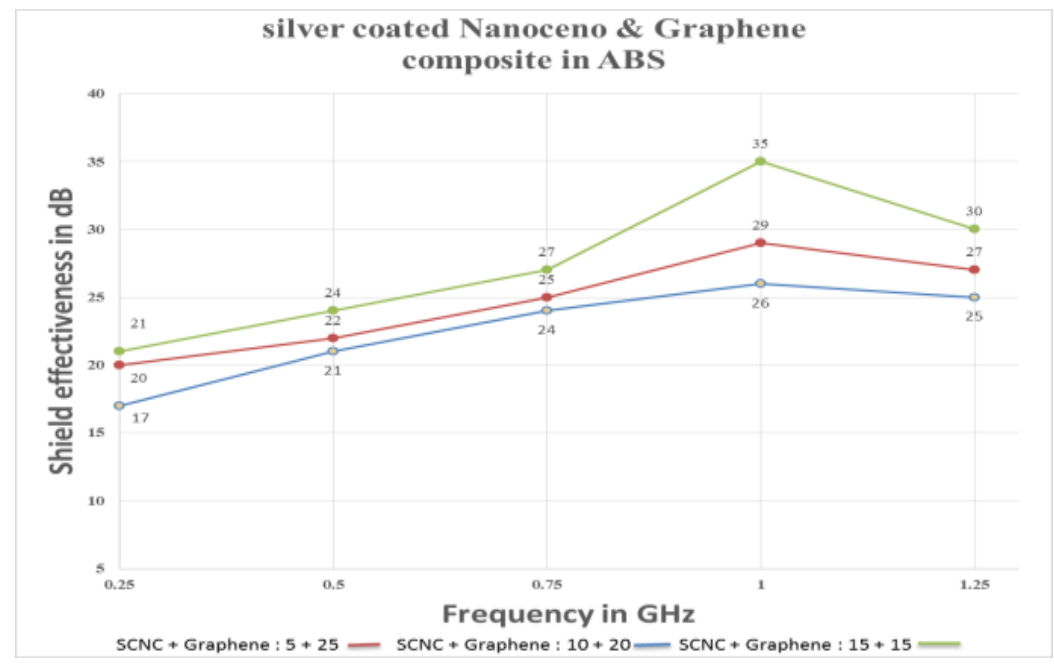

Figure 14: EMI Measurements for SCNC in ABS+Graphene Composite sheet. 
Citation: Vynatheya S, Bheema RV, Chandra SL, Sushma US (2018) Eletroless Silver Coated Nanocenosphere/Polymer Composite for EMI Shielding Effectiveness. J Material Sci Eng 7: 420. doi: 10.4172/2169-0022.1000420

Page 6 of 6

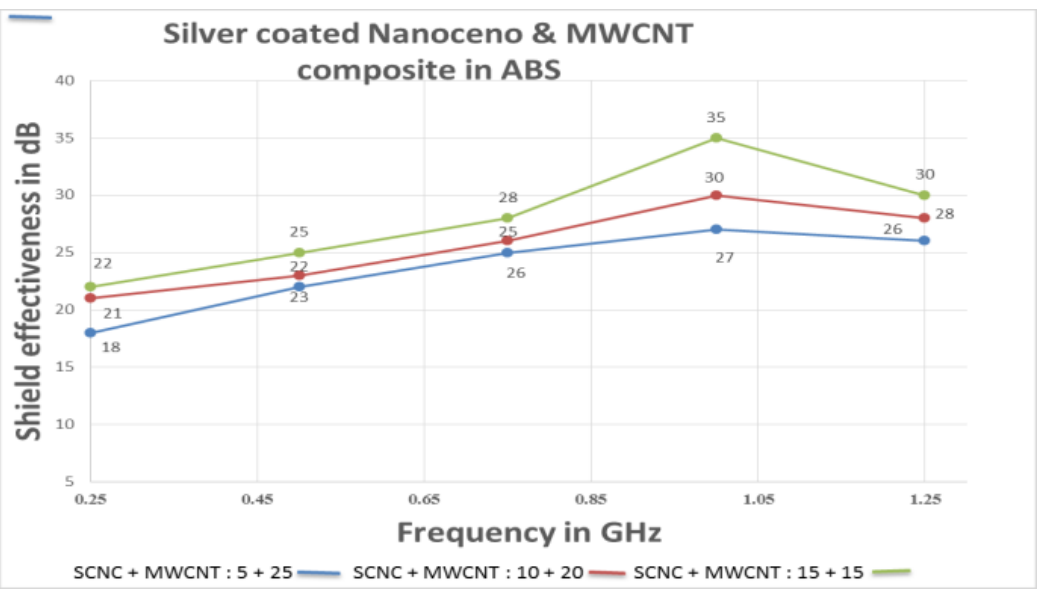

Figure 15: EMI Measurements for SCNC in ABS+MWCNT Composite sheet.

- Shielding Effectiveness is found to be appreciable at $1 \mathrm{GHz}$ for $30 \mathrm{Wt} \%$ of SCNC/ABS/Nano conducting filler composite.

\section{References}

1. Noto J, Fenical G, Tong T (2010) Automotive EMI Shielding-Controlling Automotive Electronic Emissions and Susceptibility with Proper EMI Suppression Methods. Laird Tech pp: 1-14.

2. Fomenko EV, Anshits NN, Pankova MV, Solovyov LV, Anshits AG (2011) Fly Ash Cenospheres: Composition, Morphology, Structure and Helium Permeability. World of Coal-Ash Conference, Denver, USA.
3. Agarwala RC, Agarwala V (2003) Electroless Alloy/Composite Coatings: A Review. Sadhana 28: 475-493.

4. Lu Y, Jiang S, Huang Y (2010) Ultrasonic-assisted electroless deposition of Ag on PET fabric with low silver content for EMI shielding. Surf Coat Tech 204: 2829-2833.

5. Supriya M, Sashikala M, Khodwe MS, Krishnan S, Vynatheya S (2013) Studies on the potential of silver-coated cenosphere-polycarbonate composites for electromagnetic interference shielding. International Journal of Recent Scientific Research 4: 1736-1742. 